\title{
多手性中心联萗-2-芳甲羟基-2'-醇(Ar-BINMOLs)：一类可应用于 不对称催化反应的新型手性配体
}

\author{
郑龙生 ${ }^{a}$ 宋 涛 $^{a}$ 徐利 ${ }^{*}{ }^{*, a, b}$ \\ ( ${ }^{a}$ 杭州师范大学有机硅化学及材料技术教育部重点实验室＼cjkstart杭州 311121) \\ ${ }^{b}$ 中国科学院兰州化学物理研究所羰基合成与选择氧化国家重点实验室 兰州 730000)
}

\begin{abstract}
摘要 近年来, 我们基于 1,1'-联菜基-2,2'-二酚(BINOL)为手性源成功地发展了一类多手性中心化合物联菜-2-芳甲羟 基-2'-醇(Ar-BINMOLs), 它是 BINOL 的单芐醚化合物经过不对称 [1,2]-Wittig 重排合成得到的, 涉及 $\mathrm{C}-\mathrm{O}$ 键的断裂与 新 $\mathrm{C}-\mathrm{C}$ 的构建反应, 由轴手性诱导产生新的碳手性中心, 结构独特新颖, 同时具有 $C_{2}$ 轴手性和 $\mathrm{sp}^{3}$ 碳手性的光学纯醇 酚类化合物. 目前, Ar-BINMOLs 作为新型手性配体应用于不对称催化反应已有不少的报道. 基于 BINOL 衍生物以及 近几年 Ar-BINMOLs 及其衍生物的研究进展, 重点阐述了其作为新型手性配体或手性骨架在不对称催化反应中的应用 研究进展.
\end{abstract}

关键词＼cjkstart手性配体; BINOL; 联萗-2-芳甲羟基-2'-醇(Ar-BINMOLs); [1,2]-Wittig 重排反应; 不对称催化

\section{1,1'-Binaphthalene-2- $\alpha$-arylmethanol-2'-ols (Ar-BINMOLs) with Axial and $\mathrm{sp}^{3}$-Central Chirality: A Novel Chiral Ligands for Catalytic Asymmetric Transformations}

\author{
Zheng, Longsheng ${ }^{a} \quad$ Song, Tao ${ }^{a} \quad \mathrm{Xu}$, Liwen ${ }^{* a, b}$ \\ $\left({ }^{a}\right.$ Key Laboratory of Organosilicon Chemistry and Material Technology of Ministry of Education, \\ Hangzhou Normal University, Hangzhou 310012) \\ $\left({ }^{b}\right.$ State Key Laboratory for Oxo Synthesis and Selective Oxidation, Lanzhou Institute of Chemical Physics, \\ Chinese Academy of Sciences, Lanzhou 730000)
}

\begin{abstract}
Hydroxynathalen-1-yl)naphthalene-2-ol (BINOL) and its derivatives have been used widely as chiral ligands in asymmetric catalysis. Recently, we have found that the simple axial chiral monoalkylated BINOLs could be converted into synthetically useful and enantiomerically pure 1,1'-binaphthalene-2- $\alpha$-arylmethanol-2'-ols (Ar-BINMOLs) with axial and $\mathrm{sp}^{3}$-central chirality through the axial-to-central chirality transfer of [1,2]-Wittig rearrangement. At present, the catalytic application of Ar-BINMOLs in asymmetric catalysis has been revealed by our and Yus's groups. In this account, the progress in the field of BINOL and its derivatives will also be reported concisely in the article, and the Ar-BINMOLs and its derivatives as new chiral ligands for application in asymmetric catalysis would be summarized detailedly.

Keywords chiral ligands; 1-(2-Hydroxynathalen-1-yl)naphthalene-2-ol (BINOL); 1,1'-binaphthalene-2- $\alpha$-arylmethanol2'-ols (Ar-BINMOLs); [1,2]-Wittig rearrangement; asymmetric catalysis
\end{abstract}

在过去近 30 年里, 不对称催化在有机合成化学中 一直是具有挑战性的前沿课题之一，特别是在选择性合 成与手性化学领域具有重要的地位 ${ }^{[1 ~ 3]}$. 在手性化合物 的众多合成策略中, 不对称催化合成也越来越受到人们
的关注 ${ }^{[4]}$. 与此同时, 发展高效高立体选择性的手性催 化剂在不对称合成中是一个核心难题，也显然是一个极 具应用前景的课题. 在不对称金属催化合成反应中, 手 性配体的设计与合成往往具有关键的作用 ${ }^{[5]}$. 在已报道

\footnotetext{
*E-mail: licpxulw@yahoo.com and liwenxu@hznu.edu.cn

Received March 25, 2014; revised April 17, 2014; published online May 6, 2014.

Project supported by the National Natural Science Foundation of China (No. 21173064) and the Zhejiang Provincial Natural Science Foundation of China (No. R14B030003)

国家自然科学基金(No. 21173064)和浙江省自然科学基金(No. LR14B03001)资助项目.
} 
的大量手性配体中, 具有 $C_{2}$ 对称轴的轴手性配体是一 类极具特色的配体, 在很多反应中均具有很好的立体选 择性, 充分显示其在不对称催化中具有举足轻重的地位 ${ }^{[6]}$.

自 Cram 等 ${ }^{[7]}$ 首次报道 $1,1^{\prime}$ - 联荎基-2,2'-二酚 (BINOL) 具有 $C_{2}$ 轴手性以及 Noyori 等 ${ }^{[8]}$ 首次将其应用于 酮的不对称还原以来, BINOL 及 BINOL 的衍生物作为 手性配体一直受到有机合成化学研究者们的关注. BINOL 作为理想的手性配体或手性源, 它具有较为稳定 的 $C_{2}$ 轴手性, 并且菜环骨架易于引入修饰基团, 以及二 酚可以和多种金属 Lewis 酸配位等特点. 虽然 BINOL 在不对称催化中具有不错的表现, 但是在有些反应中还 是具有一定的局限性. 因此, 研究者们越来越关注对 BINOL 的结构修饰并研究其手性化学问题, 研究表明, 通过在 3,3'位、6,6'位等引入其它基团可以改变 BINOL 的立体效应以及氧原子的电子效应，从而改变其与金属 或 Lewis 酸形成的手性络合物, 改善在反应中的立体选 择性 ${ }^{[9,10]}$. 多年来, 通过对 BINOL 的结构修饰设计新的 手性配体已经有很多报道, 在很多反应中均表现出比 BINOL 更为优异的手性诱导性能, 但是绝大部分 BINOL 衍生配体或催化剂具有一个共同点一一往往只 有 $C_{2}$ 轴手性, 或者再与另外的手性分子模块化拼接成 多手性中心的新型配体. 基于多手性中心的配体具有复 杂的空间立体环境, 以及在金属催化反应中有利于构建 优势空间构象, 近年来已被越来越多地报道并被证明多 手性中心手性配体具有一些难以预料的重要作用 ${ }^{[11 \sim 13]}$.

2011 年, 我们课题组首次报道可以高产率地高立 体选择性地合成一类多手性中心化合物, 即联菱-2-芳 甲羟基-2'-醇(Ar-BINMOLs) ${ }^{[14]}$, 它可通过 BINOL 单取 代芐醚参与的邻位酚锂活化的 [1,2]-Wittig 重排反应完 成, 它既保持 $\mathrm{BINOL}$ 的 $C_{2}$ 轴手性, 同时具有 $\mathrm{sp}^{3}$ 杂化的 碳手性中心. 我们发现将 Ar-BINMOLs 作为一类多手性 中心的新型手性配体应用于醛与乙基锌试剂的加成中, 可以得到高立体选择性的手性醇. 我们也在芳基格氏试 剂与醛的加成反应中, 通过实验证明了 Ar-BINMOLs 中 手性醇的重要性. 这表明多手性中心 Ar-BINMOLs 具有 独特性. 紧随其后, 国际上 Yus 课题组将 Ar-BINMOLs 应用于烷基铝、烷基锌、烷基镁和烷基锂与醛的加成反 应中, 都能够得到高 $e e$ 值的产物, 显示了多手性中心 Ar-BINMOLs 配体在一些催化反应中具有一定的优越 性. 基于我们在这类新型 Ar-BINMOL 分子的研究基础 上, 本文将介绍基于 BINOL 骨架合成多手性中心新型 手性配体 Ar-BINMOLs 及其衍生物的研究进展, 并总结 其在不对称催化反应中的应用性能.

\section{1 取代的 BINOL 衍生物的研究进展简况}

BINOL 具有价廉易得的优点，同时根据需要易于 引入一些大位阻或功能性基团加以结构修饰. 下面简要 介绍近年来对 BINOL 在 3 位或 3,3'位、6,6'位、4,4'位以 及 7,7'位修饰的一些代表性有机分子.

\subsection{3 位或 3,3'位取代的 BINOL}

BINOL 的 3 位或 $3,3^{\prime}$ 位很容易通过锂化反应引入修 饰基团，如对于 MOM 保护的 BINOL，通过控制有机锂 试剂的用量，加入各种亲电试剂，最后进行脱保护反应， 就可以得到各种 3 位或 3,3'位取代的产物(Scheme 1, 1 ${ }^{[15]}$. 例如, Cram 等利用 $3,3^{\prime}$ 位为 $\mathrm{Br}$ 取代的 BINOL二 甲基醚与各种芳基溴化镁在 $\mathrm{Ni}\left(\mathrm{PPh}_{3}\right)_{2} \mathrm{Cl}_{2}$ 的催化下偶联 合成 3,3'-各种取代的 BINOL，之后 Snieckus 等 ${ }^{[15 \mathrm{~d}, 16]}$ 报 道合成化合物 2 通过 Suzuki 偶联反应合成更加简便高效 (Scheme 1，2a 2 j; Scheme 2, 18). 对于杂环的引入, Suzuki 偶联产率较低, 可通过 MOM 保护的 BINOL 利

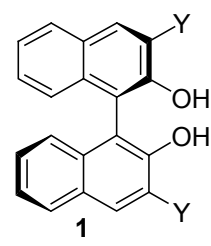

1a: $Y=F$

1b: $Y=B r$

1c: $Y=I$

1d: $Y=\mathrm{CF}_{3}$

1e: $Y=T f$

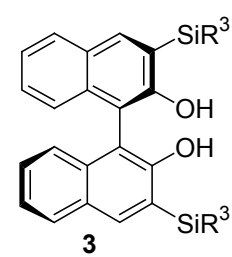

3a: $\mathrm{R}=\mathrm{Me}$ 3b: $\mathrm{R}^{3}=t-\mathrm{BuMe}_{2}$ 3c: $R^{3}=t-\mathrm{BuPh}_{2}$ 3d: $R=P h$ 3e: $\mathrm{R}=3,5-\mathrm{Me}_{2} \mathrm{C}_{6} \mathrm{H}_{3}$ 3f: $\mathrm{R}=4-\mathrm{t}-\mathrm{BuC}_{6} \mathrm{H}_{4}$

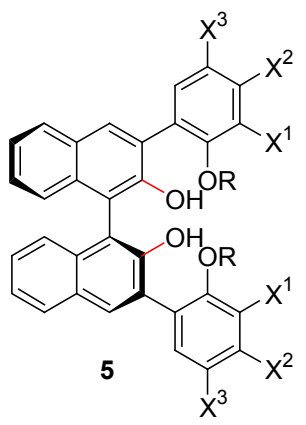

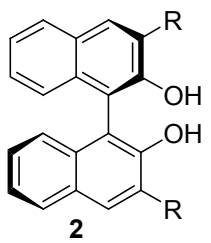

2a: $R=M e$ 2b: $\mathrm{R}=\mathrm{Ph}$

2c: $R=1-$ Naphthyl 2d: $R=2-$ Naphthyl 2e: $\mathrm{R}=3,5-t-\mathrm{Bu}_{2} \mathrm{C}_{6} \mathrm{H}_{3}$ 2f: $\mathrm{R}=3,5-\mathrm{Me}_{2} \mathrm{C}_{6} \mathrm{H}_{3}$ 2g: $\mathrm{R}=3,5-\left(\mathrm{CF}_{3}\right)_{2} \mathrm{C}_{6} \mathrm{H}_{3}$ 2h: $\mathrm{R}=4-\mathrm{MeOC}_{6} \mathrm{H}_{4}$ 2i: $R=2-P y$ 2j: $R=2-$ Thenoyl 2k: $R=2$-quinoline<smiles>[R]OC(c1ccccc1)(c1ccccc1)c1cc2ccccc2c(-c2c(O)c(C([R])(Oc3ccccc3)c3ccccc3)cc3ccccc23)c1O</smiles>

5a: $\mathrm{R}=n-\mathrm{C}_{6} \mathrm{H}_{13}, \mathrm{X}^{1}=\mathrm{X}^{2}=\mathrm{H}, \mathrm{X}^{3}=\mathrm{F}$ 5b: $\mathrm{R}=n-\mathrm{C}_{6} \mathrm{H}_{13}, \mathrm{X}^{1}=\mathrm{Me}, \mathrm{X}^{2}=\mathrm{H}, \mathrm{X}^{3}=\mathrm{F}$ 5c: $\mathrm{R}=n-\mathrm{C}_{6} \mathrm{H}_{13}, \mathrm{X}^{1}=\mathrm{X}^{2}=\mathrm{X}^{3}=\mathrm{F}$ 5d: $\mathrm{R}=n-\mathrm{C}_{6} \mathrm{H}_{13}, \mathrm{X}^{1}=\mathrm{X}^{2}=\mathrm{H}, \mathrm{X}^{3}=\mathrm{F}$ 5e: $R=M e, X^{1}=H, X^{2}=X^{3}=F$

Scheme 1 
用 $t$-BuLi 拔氢, 然后与 $\mathrm{ZnCl}_{2}$ 等形成金属锌试剂，最后 通过 $\mathrm{Pd}$ 与卤代杂环偶联得到 ${ }^{[17]} . \mathrm{Pu}$ 课题组 ${ }^{[18]}$ 通过 Suzuki 偶联反应在 3,3'位成功引入了含多个 $\mathrm{F}$ 原子的芳 环(Scheme 1, 5), 也报道多羟基化合物 4. 对于 3,3'位为 三烷基(芳基)硅基的 BINOL (Scheme 1, 3), Yamamoto 等 ${ }^{[15 \mathrm{~d}, 19,20]}$ 报道可以通过 BINOL 的硅氧烷在 $t$-BuLi 的作 用下发生硅基基团的 1,3 迁移重排得到.

Ohta 等 ${ }^{[21]}$ 报道 MOM 保护的 BINOL 经邻位锂化, 与 $\mathrm{CO}_{2}$ 反应合成得到 $3,3^{\prime}$ 位取代的羧酸 BINOL, 然后与 手性氨基醇反应可以较高收率得到 3,3'-双(2-噁唑啉 基)-1,1-联萗二酚(BINOL-Box) (Scheme 2, 6). Mancheño 等 ${ }^{[22]}$ 利用 “Click” 反应, 基于 BINOL 骨架合成多官能 团 1,2,4-三氮唑(Scheme 2, 7, 15). 3 位或 3,3'-叔胺取代的

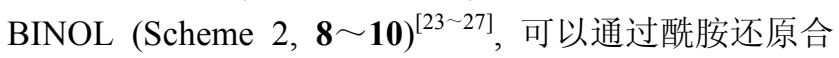
成, 也可以通过茮卤取代的 BINOL 与仲胺反应合成. $\mathrm{Li}$ 等 ${ }^{[28]}$ 通过保护的 BINOL 经邻位锂活化与 2,4,6-三氯1,3,5-三嗪反应, 然后通过胺化反应得到产率较高的 3
位或 3,3'位取代的多氮杂环(Scheme 2，11，16). Sasai 等 ${ }^{\left[{ }^{29]}\right.}$ 报道了 3 位单取代的 BINOL, 保留酚羟基作 Brønsted 酸的同时, 可以引入含氮的叔胺 (Scheme 2, 12 14)作为 Lewis 碱.

Qian 等 ${ }^{[30]}$ 利用邻位锂活化的 BINOL 与环氧化合物 开环反应，然后醚化得到 3,3 位取代的烷基醚(Scheme 3, 19). Shibasaki 和 Yoshikawa 等 ${ }^{[31]}$ 曾报道了一类新型的 3 位取代的 BINOL 桥联多酚羟基化合物(Scheme 3, 20 22), 并在一些催化合成反应中表现出优异的立体选择 性诱导性能.

上述工作充分表明了在 BINOL 的 3 位或 3,3'-进行 修饰取代是发展新型高效高立体选择性的配体较为有 效和实用的方法之一，合成方法简便，大部分产率较高， 主要是通过以手性的 BINOL 为原料, 酚羟基经保护之 后, 经过邻位锂活化, 引入各种亲电试剂, 然后对于卤 素或嗍酸可以利用 Suzuki 偶联或 $\mathrm{Cu}$ 催化偶联等方法进 一步引入其它基团.<smiles>[R]C1COC(c2cc3ccccc3c(-c3c(O)c(C4=NC([R])CO4)cc4ccccc34)c2O)=N1</smiles>

6

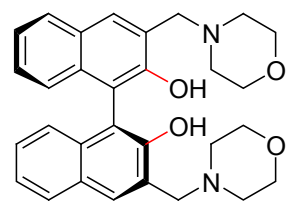

10

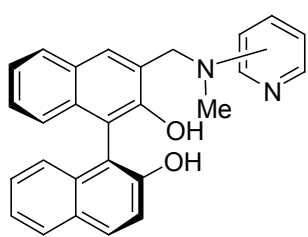

14<smiles>[R]c1cc2ccccc2c(-c2c(O)ccc3ccccc23)c1O</smiles>

18

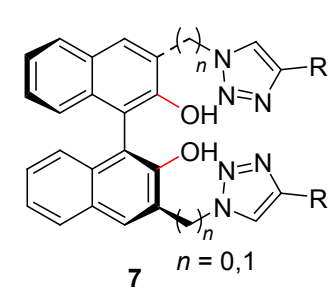

7<smiles>CN(C)c1nc(-c2cc3ccccc3c(-c3c(O)c(-c4nc(N(C)C)nc(N(C)C)n4)cc4ccccc34)c2O)nc(N(C)C)n1</smiles>

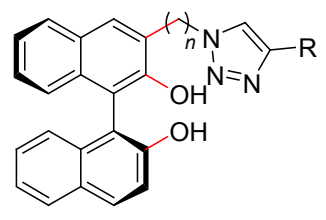

$15 n=0,1$<smiles>C[C@H](c1ccccc1)N(C)Cc1cc2ccccc2c(-c2c(O)c(CN(C)[C@H](C)c3ccccc3)cc3ccccc23)c1O</smiles>

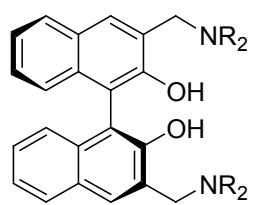

$\mathrm{R}=\mathrm{Me}, \mathrm{Et}, n-\mathrm{Pr}$<smiles></smiles>

13<smiles>Oc1c(CN2CCCC2C(O)(c2ccccc2)c2ccccc2)cc2ccccc2c1-c1c(O)c(CN2CCC[C@H]2C(O)(c2ccccc2)c2ccccc2)cc2ccccc12</smiles>

16<smiles>[R]=[Y16]n1ccnc1</smiles>

Scheme 2 


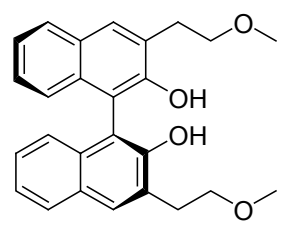

19

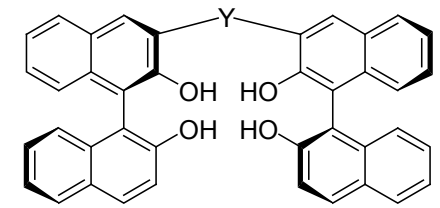

$\mathrm{Y}=\mathrm{CH}_{2},\left(\mathrm{CH}_{2}\right), \stackrel{20}{ }$

, $\mathrm{CH}_{2} \mathrm{OCH}_{2}, \mathrm{~S}, \mathrm{NMe}$

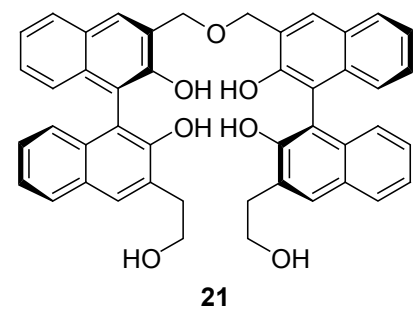

21

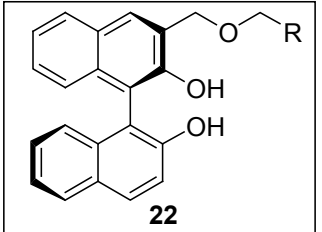<smiles>[Y6]c1cc2ccccc2c(-c2c(O)ccc3ccccc23)c1O</smiles><smiles>Oc1c(I)cccc1-c1cc(C(F)(F)F)cc(C(F)(F)F)c1</smiles><smiles>Oc1ccccc1-c1cccc(I)c1</smiles><smiles>Oc1cc2ccccc2cc1O</smiles><smiles>Cc1cc2ccccc2c(-c2cccc3ccccc23)c1O</smiles><smiles>Oc1ccccc1-c1cccc(O)c1I</smiles><smiles>Oc1cccc(-c2c3ccccc3cc3ccccc23)c1O</smiles><smiles>Oc1c(Cl)cc(Cl)cc1I</smiles><smiles>Oc1ccccc1Br</smiles>

\section{Scheme 3}

\section{$1.26,6^{\prime}$ 位、7,7'位、4,4'位取代的 BINOL}

与 3,3'位修饰的 BINOL 相比, 6,6'位取代的 BINOL 衍生物合成较为简单. 最常见的是 $6,6^{\prime}-\mathrm{Br}_{2}-\mathrm{BINOL}$, 一 般通过 BINOL 与单质溴的亲电取代就可以高收率地得 到. 基于 6,6'- $\mathrm{Br}_{2}$ - $\mathrm{BINOL}$ 进一步的修饰研究也较为成熟, 对 6,6'- $\mathrm{Br}_{2}$-BINOL 用 MOM 保护酚羟基, 经正丁基锂脱 溴, 加入其它带亲电试剂就可以得到各种卤素取代物, 如 $\mathrm{F}, \mathrm{Cl}, \mathrm{I}, \mathrm{CF}_{3}, \mathrm{C}_{2} \mathrm{~F}_{5}$ 等 ${ }^{[32]}$ (Scheme 4, 23). 6,6'- $\mathrm{Br}_{2}$-BINOL 与各种炔烃通过 Sonogashira 偶联可以得到 6,6'位各种取 代的炔烃 ${ }^{[33,34]}$ (Scheme 4, 23, 24), 也可以与各种嗍酸通 过 Suzuki 偶联反应引入其它基团 ${ }^{[35]}$. 7,7'位, 6,6'位和 7,7'
位，以及 4,4'位和 7,7'位取代的 BINOL (Scheme 4, 化合 物 25 29) $)^{[36,37,38]}$ 主要是通过两分子取代菜酚通过偶联 反应得到消旋的 BINOL 衍生物, 然后再通过手性试剂 参与的拆分过程得到, 此方法步骤较多, 合成比较繁琐.

\section{Ar-BINMOLs 的合成及其性质研究}

过去, 尽管 BINOL 的各种衍生物已被大量合成出 来，并在很多领域中得到应用，但是基于 BINOL 的 2 位 酚基引入手性中心的结构改造并不成功. 近年来，我们 课题组在这方面做了大量的研究工作, 成功地设计合成 了一类新型 $\mathrm{BINOL}$ 衍生物, 即我们称之为同时含 $C_{2}$ 轴

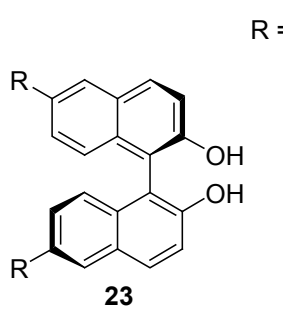<smiles></smiles>

26<smiles></smiles>

27<smiles>CN(C)CC#Cc1ccc2c(-c3c(O)ccc4cc(C#CCN(C)C)ccc34)c(O)ccc2c1</smiles>

24<smiles>Oc1ccc2ccc(Br)cc2c1-c1c(O)ccc2ccc(Br)cc12</smiles>

25<smiles></smiles>

28<smiles>Oc1cc(C(F)(F)F)cc(C(F)(F)C(F)(F)F)c1-c1c(O)cc(C(F)(F)F)c2cc(C(F)(F)F)cc(O)c12</smiles>

29

Scheme 4 
手性和 $\mathrm{sp}^{3}$ 碳手性的 Ar-BINMOLs ${ }^{[14 \mathrm{a}]}$.

\subsection{Ar-BINMOLs 的合成}

1997 年, Kiyooka 等 ${ }^{[39]}$ 报道了利用[1,2]-Wittig 反应 合成一种 Ar-BINMOLs 的研究, 但是产率较低, 反应过 程较为复杂(从 BINOL 出发合成最终产物需 5 步反应), 后处理繁琐, 且手性中心极易在后处理中发生消旋化或 $e e$ 值降低的现象, 很难应用于不对称催化过程中 (Scheme 5). [1,2]-Wittig 重排反应是经典的自由基重排 反应，在强碱的作用下，烷氧键均裂成烷基自由基和含 氧负离子的烷基自由基, 通过 1,2-烷基的迁移, 形成新 的 C-C 键的过程 ${ }^{[40]}$. 自 1942 年 Wittig 反应被首次发现 以来 ${ }^{[41]}$, 关于 Wittig 反应的机理及其在有机合成中的应 用一直备受研究者们的关注 ${ }^{[42]}$. 然而, 化学家们逐渐认 识到[1,2]-Wittig 重排反应在有机合成中具有一定的局 限性, 产率较低, 对底物的结构要求较高, Wittig 重排反 应的立体选择性控制十分困难 ${ }^{[43]}$. 因此不对称 [1,2]Wittig 重排反应很少有成功的例子.

基于[1,2]-Wittig 反应的特点, 2011 年我们利用手性 BINOL 苠醚参与的[1,2]-Wittig 重排反应(Scheme 6, 30) 一步得到了纯光学活性的多手性中心 Ar-BINMOLs (Scheme 6, 31), 反应机理如 Scheme 7 所示, 它既保持 了 BINOL 的 $C_{2}$ 轴手性, 同时新产生了 $\mathrm{sp}^{3}$ 杂化的碳手 性中心 ${ }^{[14 a, 14 b, 14 d]}$.

在此基础上, 我们也发现经过连续[1,2]-Wittig 重排
反应可合成出结构更为复杂的手性二醇, 多种类型的多 手性中心光学纯二醇被成功地合成出来 ${ }^{[14 \mathrm{~d}]}$. 例如, 利 用单取代的 BINOL 烯丙基醚经过一次 $[1,2]$-Wittig 重排 反应课高收率地得到光学纯的烯丙基取代 BINMOL 衍 生物, 然后再与烯丙基溴反应形成烯丙基醚, 经过第二 次 [1,2]-Wittig 重排反应, 可形成第二个手性醇中心, 得 到光学纯的双烯丙基 BINMOL 衍生物(Scheme 8, 37a), 两次 [1,2]-Wittig 重排反应过程均因邻位锂参与活化而 发生的完美的手性控制. 该化合物的绝对构型通过单晶 已被确定.

同样的，经过连续[1,2]-Wittig 重排反应可合成新型 Ar-BINMOLs 化合物(Scheme 9, 37b; Scheme 10, 37c).

\subsection{Ar-BINMOLs 的性质研究}

同时含轴手性和 $\mathrm{sp}^{3}$ 碳手性的 Ar-BINMOLs 具有一 些特殊的化学性质. 我们通过 ESI-MS, NMR, DSC, X单晶衍射, 圆二色谱(CD), UV 等对 Ar-BINMOLs 进行 了各种表征，显示该类化合物在手性化学上具有一定的 特殊性. 通过 ESI-MS 分析, Ar-BINMOLs 的二聚体能够 非常稳定地存在 ${ }^{[44]}$. $(R, S)-31 \mathbf{a},(S, R)-31 \mathbf{a}$ 和 $r a c-31 \mathbf{a}$ 在 ESI 阳离子质谱内检测到都有重要的二聚体中间体的存 在, 质核比 $m / z=774.97[\operatorname{dimer}+\mathrm{Na}]^{+}$, 这可能是存在分 子间氢键作用所致(Scheme 11). $(R, S)$-31a, $(S, R)-31 \mathbf{a}$ 和 rac-31a 在 ${ }^{1} \mathrm{H} \mathrm{NMR}\left(\mathrm{CDCl}_{3}\right)$ 中 Ar-BINMOLs 上的酚羟基 的质子氢显示了不同的位移，因此可以推断这是因为氢
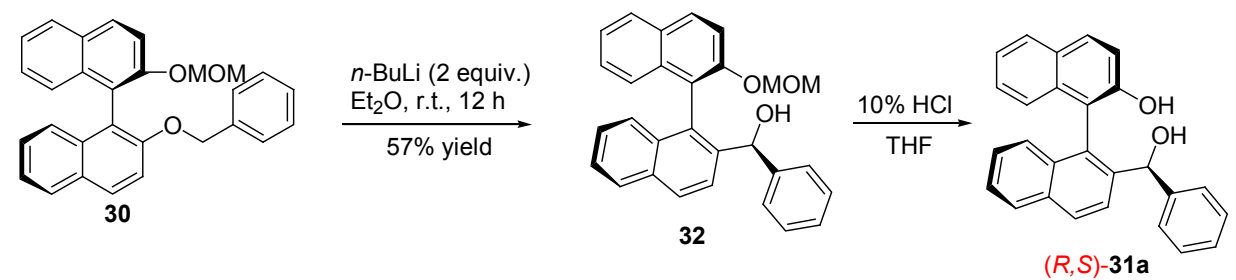

Scheme 5
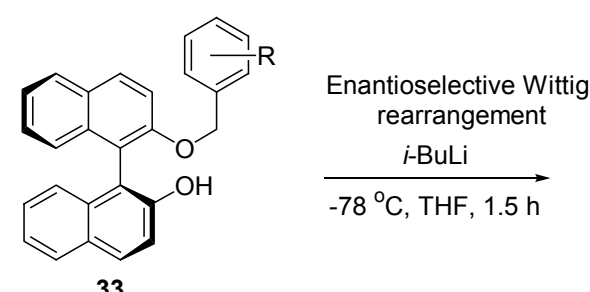

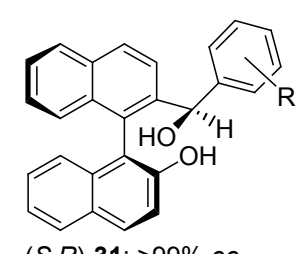

$(S, R)-31:>99 \%$ ee
$84 \% \sim 96 \%$ yield
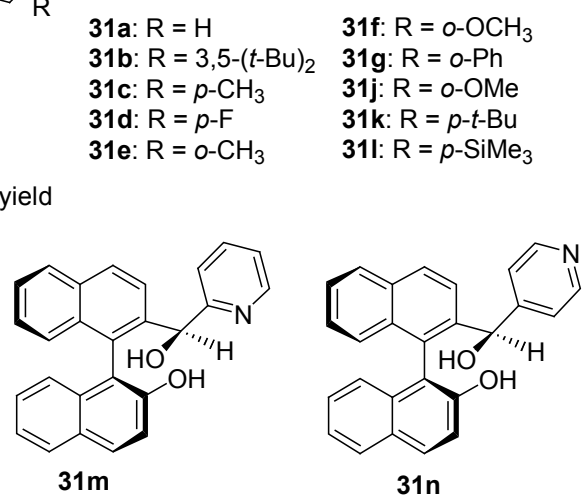

Scheme 6 

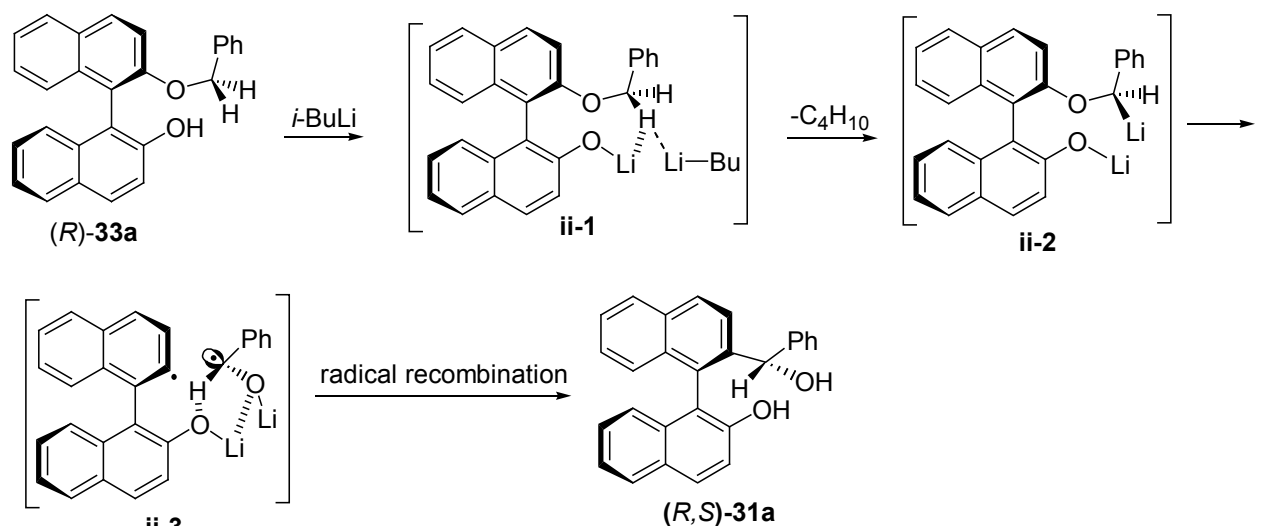

Scheme 7

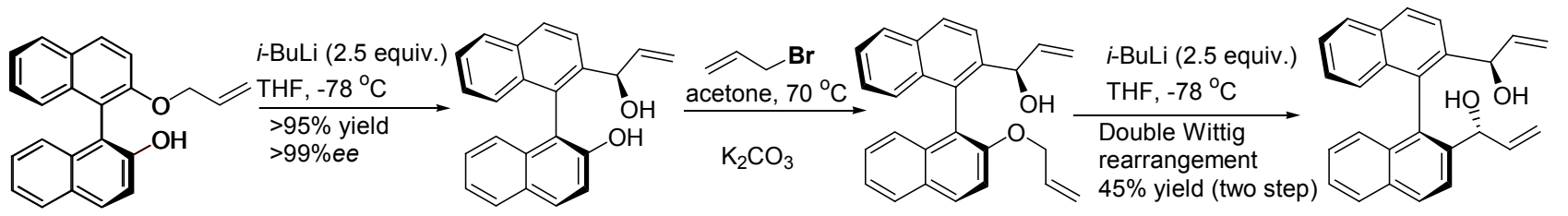

(S)-34a

$(S, R)-\mathbf{3 5 a}$

$(S, R)-36 \mathbf{a}$

$(P)-(R, R)-37 \mathbf{a}:>99 \%$ ee

Scheme 8

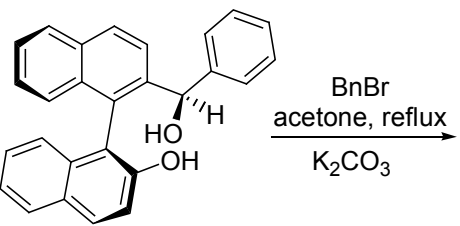

$(S, R)-31 \mathbf{a}$

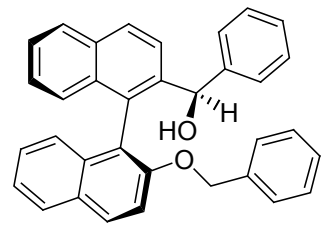

$(S, R)-\mathbf{3 6 b}$
$i$-BuLi (2.5 equiv.),

THF, $-78{ }^{\circ} \mathrm{C}$

Double Wittig

rearrangement

$40 \%$ yield (two step)

$>99 \%$ ee

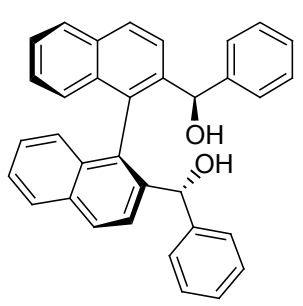

$(P)-(R, R)-\mathbf{3 7 b}$

\section{Scheme 9}

$(S, R)-31 \mathbf{a}$
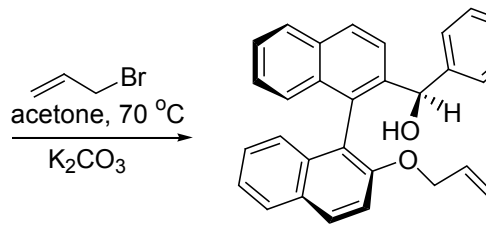

$(S, R)-36 \mathrm{c}$

$i$-BuLi (2.5 equiv.)
THF $-78^{\circ} \mathrm{C}$

作,

$35 \% \sim 40 \%$ yield

(two step)

(S,R)-35a $\frac{\stackrel{\mathrm{Ph} \sim \mathrm{Br}}{\text { acetone, } 70{ }^{\circ} \mathrm{C}}}{\mathrm{K}_{2} \mathrm{CO}_{3}}$
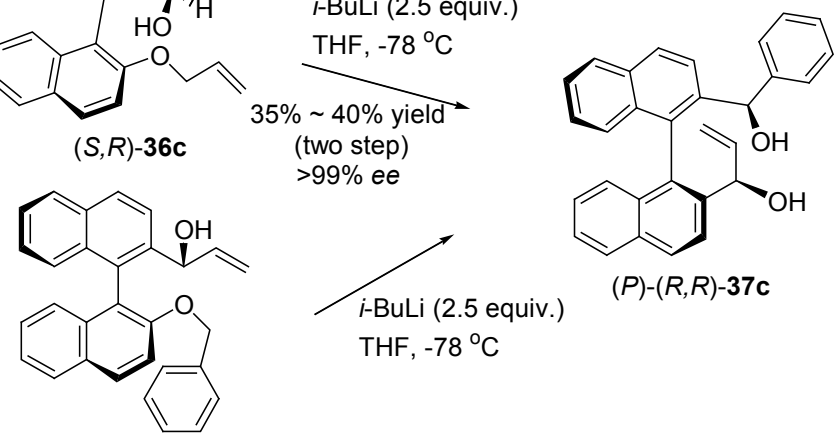

$(P)-(R, R)-\mathbf{3 7 c}$

$(S, R)-36 \mathbf{d}$

Scheme 10

键的作用和芳基的 $\pi-\pi$ 堆积作用才形成了聚合的超分子 通过 DSC 分析表明 $r a c-31 \mathrm{a}$ 表现出 Conglomerate 类分子 的特性, 这个 Conglomerate 可能是 $(R, S)$-31a 聚集体和
$(S, R)-31 \mathrm{a}$ 聚集体的混合物. 有意思的是，圆二色谱 $(\mathrm{CD})$ 显示 $r a c-31 \mathrm{a}$ 能够在固态和液态都显示出正的科顿效应, 特别在固态时有很强的光学活性(在 $230 \sim 280 \mathrm{~nm}$ 有一 


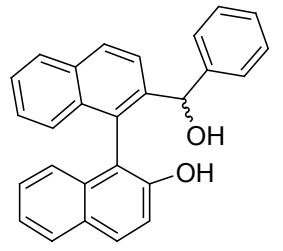

rac-31a

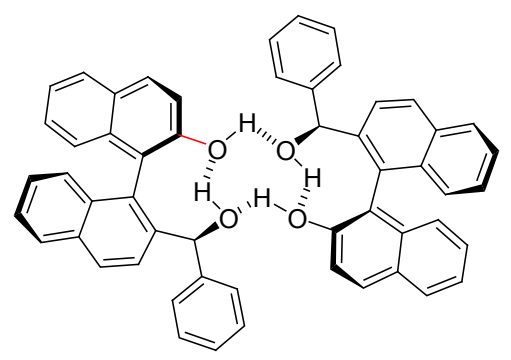

Dimer of $(R, S)-31$ a
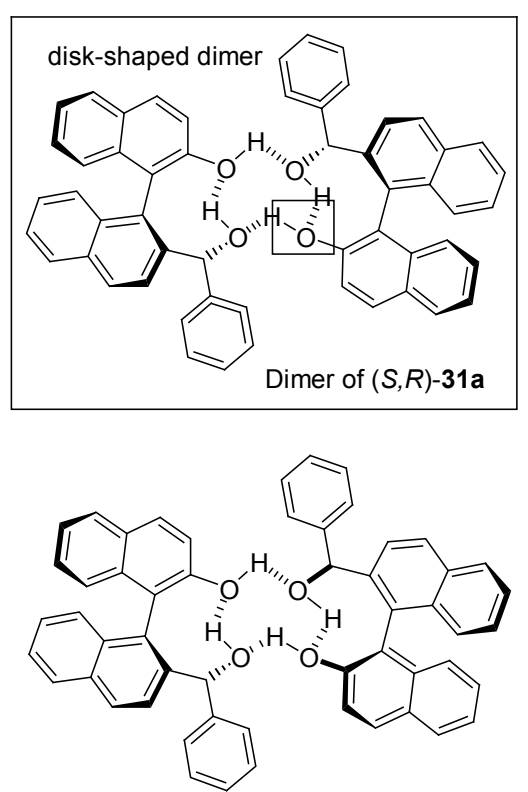

Dimer of $(S, R)-31 \mathbf{a}+(R, S)-31 \mathbf{a}(1: 1)$

Scheme 11

个很强的吸收峰), 因此可以证明 $r a c-31 \mathrm{a}$ 中存在着分子 手性的超分子聚集体. 化合物 $(S, R)-\mathbf{3 1 f}$ 的单晶 $\mathrm{X}$ 射线衍 射. 确定了 Ar-BINMOLs 的绝对构型, 同时也表明 $(S, R)-\mathbf{3 1 f}$ 分子之间沿 $C_{2}$ 轴方向观察存在芳基 $\pi-\pi$ 堆积作 用 ${ }^{[14 \mathrm{c}, 45]}$.

通过反应也可进一步支持 Ar-BINMOLs 的独特性 质. 我们发现 Ar-BINMOLs 可作为有机催化剂应用于葱 酮与反式- $\beta$-硝基烯烃的不对称加成反应中，结果表明 对映体微过量的 Ar-BINMOLs 具有手性放大效应. 对于 Ar-BINMOLs 具有轴手性和 $\mathrm{sp}^{3}$ 碳手性以及手性放大效 应, 我们认为 Ar-BINMOLs 分子间存在两种羟基的分子 间氢键作用形成二聚体超分子, 同时芳香作用可诱导聚 集形成超分子, 进而影响其在葸酮与反式- $\beta$-硝基烯烃 的不对称加成反应的催化性能.

\section{Ar-BINMOLs 在不对称催化烷基锌、烷基锂、 烷基铝和格氏试剂与醛的 1,2 加成反应中的应} 用

烷基锌与羰基的 1,2 加成反应是在构建碳-碳键的 不对称催化合成中最为常见的有机合成反应之一, 也是 目前研究较为成功的合成转化反应之一, 尤其在不对称 合成光学活性的二级醇中具有重要的价值 ${ }^{[46]}$. 自从 Ohno 等 ${ }^{[47]}$ 首次报道 $\mathrm{Ti}(\mathrm{O} i \text { - } \mathrm{Pr})_{4}$ 与手性的 bis(triflamide) 不对称催化二乙基锌与醛的 1,2 加成反应以来, 不对称 催化二乙基锌与醛的 1,2 加成反应就被广泛应用于检验 各类手性配体的立体诱导性能, 如修饰的 bis(sulfamide), TADDOLs, 和 BINOLs 等手性配体的催化活性 ${ }^{[48]}$.
Ar-BINMOLs 是 BINOL 的衍生物, 具有 BINOL 的 $C_{2}$ 轴手性和 $\mathrm{sp}^{3}$ 杂化的碳手性的醇酚类化合物. 为了研究 Ar-BINMOLs 作为手性配体的催化活性, 我们课题组首 次将其应用于 $\mathrm{Ti}(\mathrm{O} i-\mathrm{Pr})_{4}$ 催化二乙基锌与芳香醛的 1,2 加成反应中. 从表 1 可知，当配体为 $(S, R, S)-\mathbf{3 1 i}$ 时，该反 应能够得到很好的产率和最高为 $99.9 \%$ 的 $e e$ 值, 底物也 具有很好的适应性 ${ }^{[14 c]}$.

表 1 Ar-BINMOL 31i 催化醛与二乙基锌的加成 Table 1 Addition of diethylzinc to aldehydes using Ar- BINMOL 31i

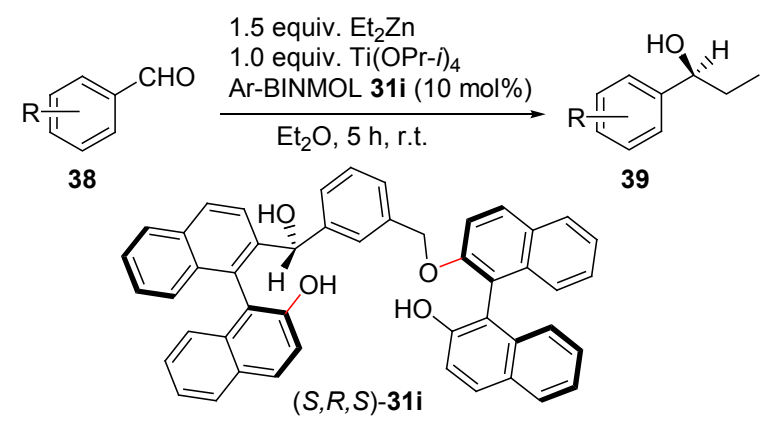

\begin{tabular}{clcc}
\hline Entry & \multicolumn{1}{c}{$\mathrm{R}$} & Yield $/ \%$ & $e e / \%$ \\
\hline 1 & $m-\mathrm{Me}$ & 92 & 97 \\
2 & $m-\mathrm{Br}$ & 94 & $>99.9$ \\
3 & $m-\mathrm{Cl}$ & 95 & 97 \\
4 & $p-\mathrm{OMe}$ & 91 & 97 \\
5 & $p-\mathrm{Cl}$ & 93 & 97 \\
6 & $p-\mathrm{Me}$ & 91 & 97 \\
7 & $p-\mathrm{F}$ & 90 & 98 \\
8 & $o-\mathrm{F}$ & 89 & 99 \\
9 & $m-\mathrm{F}$ & 94 & 99 \\
10 & $3,4-\mathrm{F}$ & 92 & 96 \\
\hline
\end{tabular}


毫无疑问, Ar-BINMOLs 作为新型手性配体在不对 称催化二乙基锌与芳香醛的 1,2 加成中具有很好的催化 活性. 为了进一步研究 Ar-BINMOLs 的催化活性, 同时 也为合成廉价的高对映体选择性的二级醇提供有效方 法，我们选择芳基格氏试剂和甲基格氏试剂与芳香醛的 不对称 1,2-加成反应作为模型反应. 在芳基格氏试剂与 芳基溴化镁的不对称 1,2 加成反应中, 经过对常见的 ArBINMOL 手性配体进行篎选, 发现最佳配体为 $(S, R)$ 31h, 可得到 $72 \% \sim 92 \%$ 的产率和最高为 $72 \%$ 的 $e e$ 值(表 $2)^{[14 \mathrm{~d}]}$. 为了验证 Ar-BINMOLs 中碳手性的特殊性, 我们 还进一步设计了另外两种手性配体进行对比, 结果表明 醇羟基具有重要的作用, 并推测了与 $\mathrm{Ti}(\mathrm{O} i-\mathrm{Pr})_{4}$ 作用可 能存在的中间过渡态(图 1$)^{[14 \mathrm{~d}]}$.

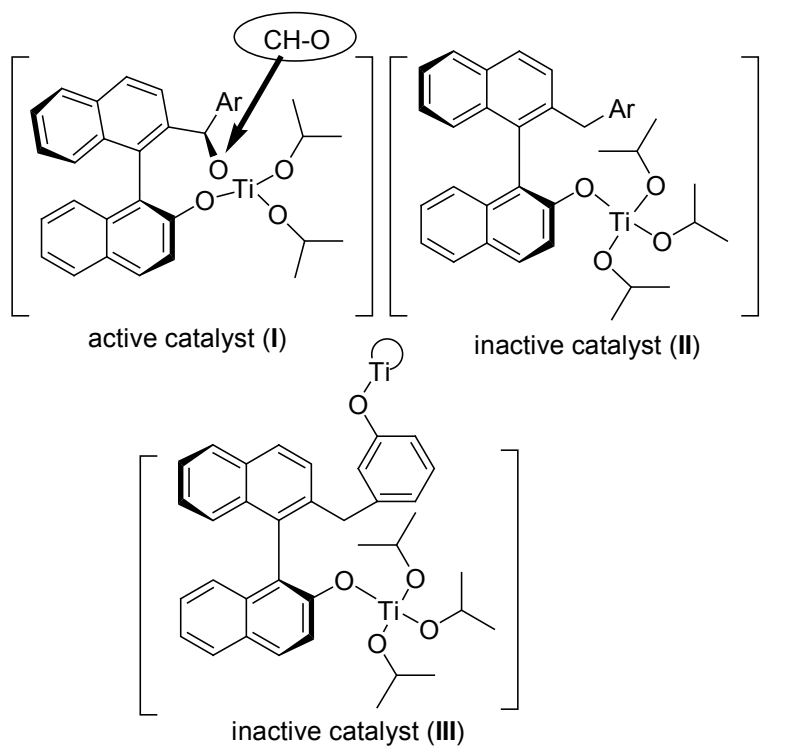

图 1 Ar-BINMOL 及其衍生物与钣可能存在的过渡态模型 (Ar-BINMOL 中醇羟基具有重要作用)

Figure 1 Possible titanium complex derived from Ar-BINMOL (the importance of the alcohol)

自从我们课题组报道 Ar-BINMOLs 的合成及应用 以来, Yus 课题组 ${ }^{[49]}$ 也相继研究了 Ar-BINMOLs 作为手 性配体的应用研究, 成功地将其应用于不对称催化烷基 锂与芳香醛的 1,2 加成反应、烷基铝与醛的 1,2 加成反 应、烷基格氏试剂与醛的 1,2 加成反应. 如不对称催化 烷基锂与芳香醛的 1,2 加成反应，最佳配体为 $(S, R)-31 \mathbf{a}$, 得到最高为 $96 \%$ 的 $e e$ 值; 同样的配体, 在不对称催化烷 基铝与醛的 1,2 加成反中可得到最高为 $99 \%$ 的 $e e$ 值; Yus 等 ${ }^{\left[{ }^{[9]}\right.}$ 还发现该配体同样可以应用于不对称催化烷基格 氏试剂与芳香醛的 1,2 加成反应, 可得到很好的立体选 择性，产物的 $e e$ 值最高可以达到 $96 \%$ ，而烷基格氏试剂 与脂肪醛 1,2 加成反应, 最佳配体为 $(S, R)-\mathbf{3 1} \mathbf{n}$, 其立体 诱导性能更为理想, 针对很多底物可得到最高为 $99 \%$ 的
表 2 Ar-BINMOL 31h 催化醛与格氏试剂的加成

Table 2 Addition of Grignard reagents to aldehydes using Ar-BINMOL 31h

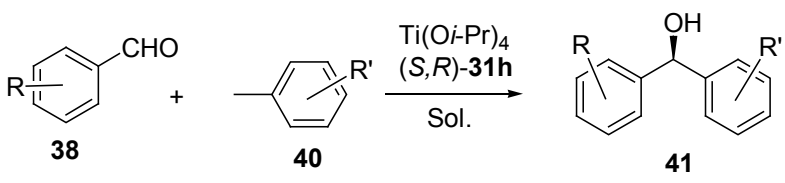

\begin{tabular}{cllcc}
\hline Entry & \multicolumn{1}{c}{$\mathrm{R}$} & \multicolumn{1}{c}{$\mathrm{R}^{\prime}$} & Yield/\% & $e e / \%$ \\
\hline 1 & $o-\mathrm{CH}_{3}$ & $\mathrm{H}$ & 84 & 70 \\
2 & $m-\mathrm{OCH}_{3}$ & $\mathrm{H}$ & 91 & 55 \\
3 & $m-\mathrm{F}$ & $\mathrm{H}$ & 91 & 54 \\
4 & $m-\mathrm{Br}$ & $\mathrm{H}$ & 92 & 50 \\
5 & $p-\mathrm{OCH}$ & $\mathrm{H}$ & 70 & 61 \\
6 & $\mathrm{Naphthyl}_{3}$ & $\mathrm{H}$ & 77 & 54 \\
7 & $o-\mathrm{CH}_{3}$ & $\mathrm{~F}$ & 74 & 65 \\
8 & $o-\mathrm{OCH} \mathrm{H}_{3}$ & $\mathrm{~F}$ & 83 & 69 \\
9 & $m-\mathrm{Br}$ & $\mathrm{F}$ & 76 & 56 \\
10 & $m-\mathrm{F}$ & $\mathrm{F}$ & 82 & 54 \\
11 & $o-\mathrm{CH}$ & $p$-OMe & 77 & 72 \\
12 & $o-\mathrm{Cl}$ & $p$-OMe & 72 & 66 \\
13 & $\mathrm{H}$ & $p$-OMe & 89 & 69 \\
14 & $m-\mathrm{Br}$ & $p$-OMe & 70 & 71 \\
\hline
\end{tabular}

$e e$ 值, 反应过程中配体 $(S, R)-\mathbf{3 1} \mathbf{n}$ 与钛催化剂可能存在的 过渡态为 III 和 IV(图 2). 因此, 利用 Ar-BINMOLs 作为 手性配体可应用不对称催化烷基金属试剂与醛的 1,2 加 成反应，而且已有工作表明都能得到高对映体选择性的 产物，为获取各种光学活性的手性醇提供良好的催化剂

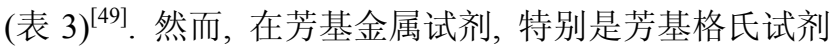
和芳香醛的 1,2 加成反应中, 其立体选择性有待进一步 改善，因此发展多样性的 Ar-BINMOLs 衍生物有望提高 其立体选择性.

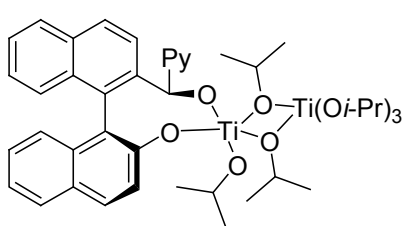

III

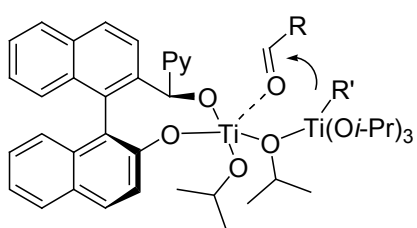

IV
图 2 催化体系中涉及的可能过渡态

Figure 2 Possible intermediates involved in the catalysis

\section{4 基于手性 Ar-BINMOLs 骨架的衍生配体合 成及其在不对称催化中的应用}

4.1 基于手性 Ar-BINMOLs 骨架 Salan 配体的合成及 其在不对称催化中的应用

Salan 配体是一类特殊的手性配体并可广泛应用于 各类有机合成反应中. 最近, 我们 ${ }^{[50]}$ 设计合成了一类手 性 Ar-BINMOL 衍生的的 Salan-47 配体(Scheme 12), 并 对 Salan-47 配体和 Salan-47 配体与金属盐进行紫外可见 
表 3 Ar-BINMOL 31a, 31h 或 31n 催化醛与格氏试剂的加成 Table 3 Addition of alkyllithium reagents, organo-aluminum reagents, Grignard reagents to aldehydes using Ar-BINMOL 31a, 31h or 31n

\begin{tabular}{|c|c|c|}
\hline Aldehyde & $\begin{array}{l}\text { Organometallic } \\
\text { reagent }\end{array}$ & Yield/\% $(e e / \%)$ \\
\hline \multirow{3}{*}{$\mathrm{PhCHO}$} & $\mathrm{MeLi}^{[49 b]}$ & $87(90)$ \\
\hline & $\mathrm{Me}_{3} \mathrm{Al}^{[49 \mathrm{c}]}$ & $99(94)$ \\
\hline & $\mathrm{MeMgBr}^{[14 \mathrm{~d}, 49 \mathrm{a}]}$ & $92(88)$ or $96(85)$ \\
\hline \multirow{3}{*}{$p-\mathrm{MeOC}_{6} \mathrm{H}_{4} \mathrm{CHO}$} & $\mathrm{MeLi}^{[49 b]}$ & $91(89)$ \\
\hline & $\mathrm{Me}_{3} \mathrm{Al}^{[49 \mathrm{c}]}$ & $87(94)$ \\
\hline & $\mathrm{MeMgBr}^{[14 \mathrm{~d}]}$ & $95(80)$ \\
\hline \multirow{3}{*}{$p-\mathrm{ClC}_{6} \mathrm{H}_{4} \mathrm{CHO}$} & $\mathrm{MeLi}^{[49 b]}$ & $74(84)$ \\
\hline & $\mathrm{Me}_{3} \mathrm{Al}^{[49 c]}$ & $92(94)$ \\
\hline & $\mathrm{MeMgBr}^{[14 \mathrm{~d}, 49 \mathrm{a}]}$ & $98(84)$ or $92(90)$ \\
\hline \multirow{3}{*}{ 2-Naphthyl-CHO } & $\mathrm{MeLi}^{[49 b]}$ & $85(90)$ \\
\hline & $\mathrm{Me}_{3} \mathrm{Al}^{[49 c]}$ & 99 (94) \\
\hline & $\operatorname{MeMgBr}{ }^{[14 d, 49 a]}$ & $92(90)$ or $95(92)$ \\
\hline \multirow{3}{*}{ 2-Thienyl-CHO } & $\mathrm{MeLi}^{[49 b]}$ & $57(88)$ \\
\hline & $\mathrm{Me}_{3} \mathrm{Al}^{[49 \mathrm{c}]}$ & $68(80)$ \\
\hline & $\mathrm{MeMgBr}^{[49 \mathrm{a}]}$ & $53(58)$ \\
\hline \multirow{3}{*}{$\mathrm{PhCH}_{2} \mathrm{CHO}$} & $\mathrm{MeLi}^{[49 b]}$ & $23(62)$ \\
\hline & $\mathrm{Me}_{3} \mathrm{Al}^{[49 \mathrm{c}]}$ & $92(84)$ \\
\hline & $\mathrm{MeMgBr}^{[49 \mathrm{a}]}$ & $70(70)$ \\
\hline \multirow{3}{*}{$t$-Bu-CHO } & $\mathrm{MeLi}^{[49 b]}$ & $<2$ (nd) \\
\hline & $\mathrm{Me}_{3} \mathrm{Al}^{[49 \mathrm{c}]}$ & $55(99)$ \\
\hline & $\mathrm{MeMgBr}^{[49 \mathrm{a}]}$ & $58(99)$ \\
\hline \multirow{3}{*}{$\mathrm{PhCHO}$} & $n-\mathrm{BuLi}^{\mathrm{i49b}]}$ & $78(92)$ \\
\hline & $\mathrm{Et}_{3} \mathrm{Al}^{[49 \mathrm{c}]}$ & $77(90)$ \\
\hline & $\mathrm{EtMgBr}^{[49 \mathrm{a}]}$ & $95(86)$ \\
\hline \multirow{3}{*}{$\mathrm{PhCHO}$} & $n-\mathrm{BuLi}^{\mathrm{i49b}]}$ & $90(96)$ \\
\hline & $i-\mathrm{Bu}_{3} \mathrm{Al}^{[49 \mathrm{c}]}$ & - \\
\hline & $n-\mathrm{BuMgBr}^{[49 \mathrm{a}]}$ & $90(96)$ \\
\hline \multirow{3}{*}{$p-\mathrm{MeOC}_{6} \mathrm{H}_{4} \mathrm{CHO}$} & $n-\mathrm{BuLi}^{[49 \mathrm{~b}]}$ & $89(94)$ \\
\hline & $n-\mathrm{Pr}_{3} \mathrm{Al}^{[49 \mathrm{c}]}$ & $26(92)$ \\
\hline & $n$-BuMgBr & $81(92)$ \\
\hline \multirow{3}{*}{ 2-Naphthyl-CHO } & $\mathrm{PhLi}^{[49 b]}$ & $96(17)$ \\
\hline & $\mathrm{Ph}_{3} \mathrm{Al}^{[49 \mathrm{c}]}$ & $90(20)$ \\
\hline & $\mathrm{PhMgBr}{ }^{[49 a]}$ & $98(15)$ \\
\hline \multirow{3}{*}{ Cyclohexyl-CHO } & $\mathrm{PhLi}^{[49 b]}$ & $92(39)$ \\
\hline & $i-\mathrm{Bu}_{3} \mathrm{Al}^{[49 \mathrm{c}]}$ & 一 \\
\hline & $n-\mathrm{BuMgBr}^{[49 \mathrm{a}]}$ & $98(50)$ \\
\hline Octyl-CHO & & $98(88)$ \\
\hline $\mathrm{PhCH}_{2} \mathrm{CH}_{2} \mathrm{CHO}$ & & $81(86)$ \\
\hline Cyclohexyl-CHO & $\mathrm{MeMgBr}^{[49 \mathrm{~d}]}$ & $61(92)$ \\
\hline$(E)-\mathrm{PhCH}=\mathrm{CHCHO}$ & & $>99(82)$ \\
\hline $\mathrm{PhC} \equiv \mathrm{CCHO}$ & & $80(60)$ \\
\hline
\end{tabular}

光谱、荧光光谱等物性测试. 通过其与各种金属盐的苂 光分析测试, 我们发现 Salan-47 配体与 $\mathrm{CuCl}$ 的苂光光 谱信号最强, 因此该类配体在一定程度上可作为荧光探
针应用于 $\mathrm{Cu}(\mathrm{I})$ 离子的检测中 ${ }^{[51]}$. 基于一些物性测试分 析数据, 我们将 Salan-47 配体与 $\mathrm{Cu}$ 的络合物作为催化 剂应用于 Henry 反应, 有意思的是, 该类 Salan- $47-\mathrm{Cu}$ 催 化剂对不同结构的醛具有特异性区分效应. 研究结果表 明对未取代的苯甲醛及含卤素的芳香醛具有较好的反 应结果, $e e$ 值可高达 $91 \%$, 产率有较为理想(Eq. 1). 然而 对甲基、甲氧基或硝基取代的芳香醛，该类 Henry 反应 几乎不发生，甚至脂肪醛也没有反应活性，这些意外的 结果促使我们对 Salan-铜催化剂进行了一些研究, 初步 的结构分析表明 Salan 配体的茮基形成了较大的立体位 阻, 可能形成了可识别某些醛的空穴, 但尚需进一步的 证据来支撑其独特的空间结构.

\section{2 基于手性 Ar-BINMOLs 骨架单膦配体(Ar-NNPs) 的合成及其在不对称催化中的应用}

手性膦配体在过渡金属催化中具有极为重要的地 位，在过去几十年里已被证明是最有价值的一类配体. 基于 Ar-BINMOLs 骨架中的 $C_{2}$ 轴手性, 我们 ${ }^{[52]}$ 设计合 成了一类新型手性单膦配体(Ar-NNPs), 并将其应用于 银催化的不对称 2-(三甲基硅氧基)呋喃与亚胺的 Mannich 插烯反应(Eq. 2). 研究表明银与手性单膦配体 Ar-NNPs 形成的复合物可有效催化此类 Mannich 插烯 反应，亚胺底物的适应性较好，均能够得到很好的分离 产率(最高为 $99 \%$ ), 而且该类配体的立体选择性较好(最 高为 $78 \% e e)$, 非对映异构选择性很高 $(d r>99: 1)$.

通过 X 单晶衍射分析, 银与手性单膦配体 Ar-NNPs 形成的配合物中手性单膦配体 Ar-NNPs 的芐基具有双 重作用, $\mathrm{Ag}-\pi / \pi-\pi$ 弱堆积作用和立体位阻的排斥作用有 利于 2-(三甲基硅氧基)呋喃形成的亲核试剂从 $R e$ 面对 亚胺进行加成, 从而保证了该类单膦配体具有高立体选 择性诱导性能. 值得一提的是，该类配体没有其它官能 团，仅靠单膦中心与茮基的芳香作用获得较为理想的立 体选择性诱导能力, Yamamoto 等 ${ }^{[53]}$ 对本工作给予了积 极的评论并认为在该反应中单膦配体 Ar-NNPs 的芐基 不仅有利于形成银 $-\pi / \pi-\pi$ 堆积作用，而且其明显的立体 位阻效应也保证了 2-(三甲基硅氧基)呋喃对亚胺的高立 体选择性加成.

基于 Ar-BINMOLs 骨架，我们还成功合成了一类含 希夫碱膦配体 55 (Eq. 3) ${ }^{[54]}$. 虽然在有机锌与烯酮的 1,4 加成反应中没有表现出良好的催化活性，但是该工作对 多手性中心膦配体 HZNU-Phos 的构建及其催化反应研 究具有一定的启示作用 ${ }^{[54]}$.

\section{5 结论与展望}

综上所述，同时具有 $C_{2}$ 轴手性和 $\mathrm{sp}^{3}$ 碳手性的多手 性中心 Ar-BINMOLs, 合成方法简便, 产率高, 具有很 


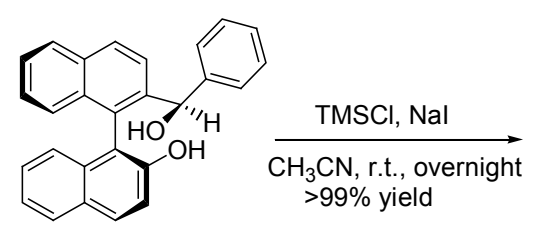

Ar-BINMOL

$(S, R)-31 \mathbf{a}$

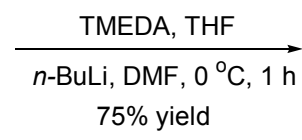

$75 \%$ yield

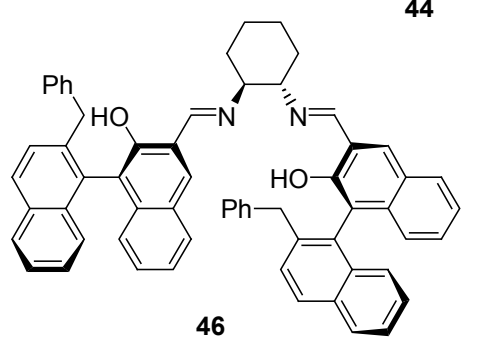

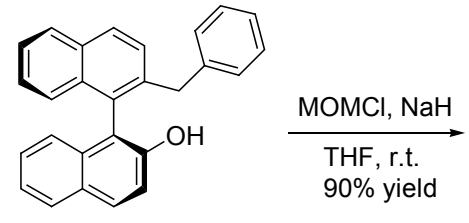

42a

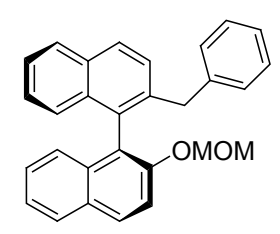

43

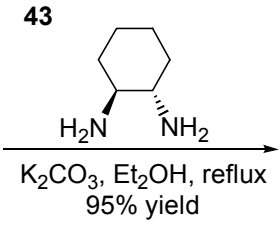

$\underset{0{ }^{\circ} \mathrm{C}, 1 \mathrm{~h}}{\stackrel{\text { THF, Con. } \mathrm{HCl}}{\longrightarrow}}$<smiles>[15CH3]c1cccc2c(-c3c(Cc4ccccc4)ccc4ccccc34)c(O)c(C=O)cc12</smiles>

95\% yield $95 \%$ yield 45

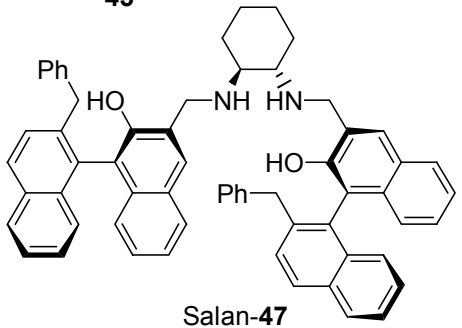

$\underset{\mathrm{MeOH}, 0{ }^{\circ} \mathrm{C}}{\stackrel{\mathrm{NaBH}_{4}}{\longrightarrow}}$ $>99 \%$ yield

Salan-47

Scheme 12

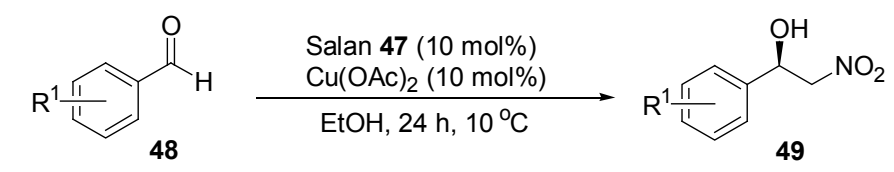

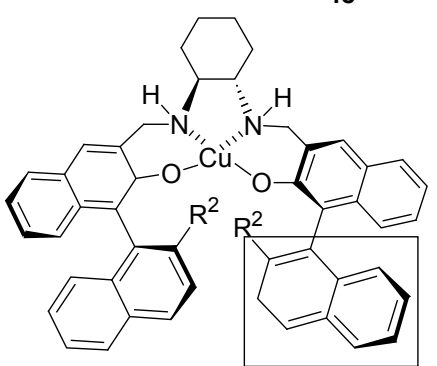

Salan-47-Cu
$\mathrm{R}^{2}=$<smiles>c1ccccc1</smiles>

, $95 \%$ yield, $91 \%$

$\mathrm{R}=2-\mathrm{F}, 99 \%$ yield, $59 \%$ ee

$\mathrm{R}=3-\mathrm{F}, 92 \%$ yield, $89 \%$ ee

$\mathrm{R}=4-\mathrm{F}, 98 \%$ yield, $79 \%$ ee

$\mathrm{R}=2-\mathrm{Cl}, 92 \%$ yield, $58 \%$ ee

$\mathrm{R}=3-\mathrm{Cl}, 90 \%$ yield, $81 \%$ ee

$\mathrm{R}=2-\mathrm{Br}, 90 \%$ yield, $76 \%$ ee

$\mathrm{R}=4-\mathrm{Br}, 91 \%$ yield, $83 \%$ ee

$\mathrm{R}=$ other groups: $\mathrm{Me}, \mathrm{OMe}$,

$\mathrm{NO}_{2}, \mathrm{OH}$, etc.

no or trace product $(<5 \%$ yield)
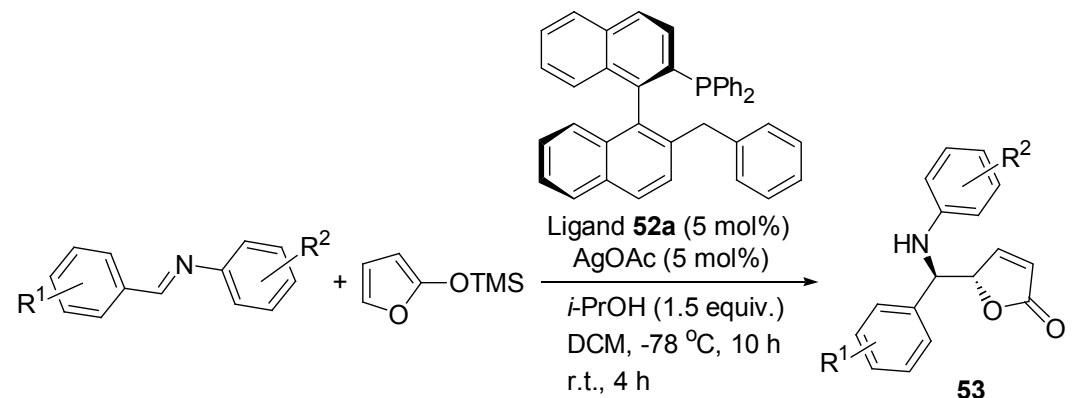

up to $99 \%$ yield

up to $78 \%$ ee, $>99: 1 d r$

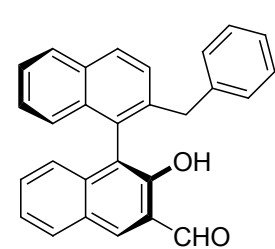

45

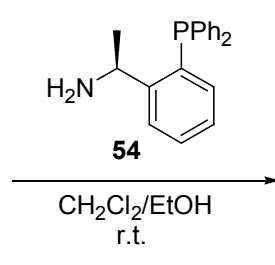

r.t.<smiles>C[C@H](N=Cc1cc2ccccc2c(-c2c(Cc3ccccc3)ccc3ccccc23)c1O)c1ccccc1P(C)(C)(C)C</smiles> 
好的应用价值. 而且该类手性分子易于进一步修饰或进 行结构改造, 可对 Ar-BINMOLs 剩余的酚羟基进一步芐 基化或烯丙基化，通过二次 [1,2]-Wittig 重排可以构建含 $C_{2}$ 轴手性和两个 $\mathrm{sp}^{3}$ 碳手性的 Ar-BINMOLs. 在短短的 3 年里, Ar-BINMOLs 已被成功地作为新型手性配体应用 于有机锌试剂、有机锂试剂、有机铝试剂以及格氏试剂 与醛的 1,2 加成反应等重要有机转化反应, 其中上述烷 基或芳基金属试剂和醛的加成能够得到高 $e e$ 值的手性 醇. Ar-BINMOLs 也可以作为良好的手性骨架应用于 Salan 配体、单膦配体、席夫碱配体等设计中, 在 Henry 反应、Mannich 插烯反应等取得了较好的结果. 我们相 信含有 $C_{2}$ 轴手性和 $\mathrm{sp}^{3}$ 碳手性的 Ar-BINMOLs 将能够在 越来越多的有机合成反应中表现出具有很好的催化活 性和立体诱导性能, 陆续支持 Ar-BINMOLs 及其衍生物 作为优异手性配体的强有力证据将会在后续研究报道 中不断出现 ${ }^{[5]}$, 有望成为一类具有特殊性能的特色配 体并应用于各类不对称催化反应中. 同时我们也希望更 多的有机化学工作者能够把这类手性骨架用在化学研 究的各个领域之中, 相信将会有一些有意义的新发现与 新用途不断被揭示出来.

\section{作者简介}

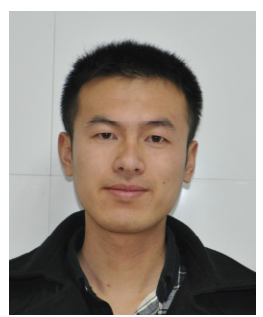

郑龙生, 在读硕士研究生. 1988 年生于浙江省瞿州 市, 2011 年浙江大学宁波理工学院本科毕业, 同年进入 杭州师范大学有机硅化学及材料技术教育部重点实验 室加入徐利文课题组攻读有机化学硕士学位. 研究方向 为手性配体的设计与合成, 金属导向的不对称催化反 应, 在国际重要学术期刊已发表学术论文 5 篇.

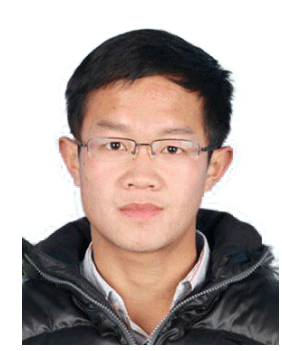

宋涛, 在读硕士研究生. 1990 年生于安徽省安庆市, 2012 年安庆师范学院本科毕业, 同年进入杭州师范大 学有机硅化学及材料技术教育部重点实验室加入徐利
文课题组攻读有机化学硕士学位. 研究方向为手性配体 的设计与合成，金属导向的不对称催化反应，目前已在 国际重要学术期刊已发表学术论文 2 篇.

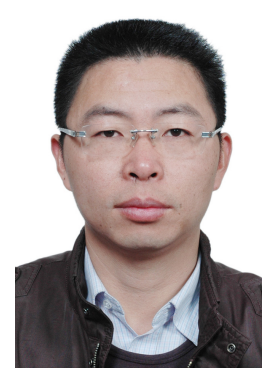

徐利文, 1976 年生于安徽省黄山市, 1998 年毕业于 安徽师范大学化学系(理学学士), 2004 年毕业于中国科 学院兰州化学物理研究所, 获中国科学院研究生院理学 博士学位. 先后在中国科学院兰州化学物理研究所(夏 春谷课题组, 副研究员, 2004 2006 年)、杭州师范大学 (课题组长, 2006 年至今)工作, 期间曾于法国国家科研 中心勒芒大学 Mortier 课题组(2005 2006 年)、新加坡 国立大学化学系 Lu Yixin 课题组(Research Fellow, 2007 2008 年)、东京大学药学院 Shibasaki 课题组(JSPS 特别研究员, 2008 2009 年)从事合作研究工作. 2009 年 任杭州师范大学有机硅化学及材料技术教育部重点实 验室研究员, 2011 年起先后被评为浙江大学博士生导师 (兼), 中国科学院兰州化学物理研究所羰基合成与选择 性氧化国家重点实验室客座教授，博士生导师，先后被 遴选为政府特殊津贴获得者, 浙江省高校中青年学科带 头人. 主要从事不对称催化合成、均相催化及有机硅化 学等方面的研究, 在国内外 SCI 学术期刊发表论文 120 余篇(包括 ESI top 1\%高被引论文 6 篇), 已被他人引用 超 2000 次; 参与国外学术专著撰写 3 章, 获授权发明专 利 5 项.

\section{References}

[1] Ager, D. J.; East, M. B. Asymmetric Synthetic Methodology, CRC, Boca Raton, FL, 1996.

[2] Ojima, I. Catalytic Asymmetric Synthesis, John Wiley \& Sons, New Jersey, 2004.

[3] Gawley, R. E.; Aubé, J. Principles of Asymmetric Synthesis, 2nd ed, Elsevier, 2012.

[4] Ding, K. L.; Han, Z. B.; Wang, Z. Chem. Asian J. 2009, 4, 32.

[5] Yong, T. P.; Jacobsen, E. N. Science 2003, 299, 1691.

[6] Kagan, H. B. In Asymmetric Synthesis, Ed.: Morrison, J. D., Academic Press, New York, 1985.

[7] Cram, D. J.; Cram, J. M. Acc. Chem. Res. 1978, 11, 8.

[8] Noyori, R.; Tomino, I.; Tanimoto, Y. J. Am. Chem. Soc. 1979, 101, 3129.

[9] Chen, Y.; Yekta, S.; Yudin, A. K. Chem. Rev. 2003, 103, 3155.

[10] Brunel, J. M. Chem. Rev. 2007, 107, 1.

[11] Kamikawa, K.; Watanabe, T.; Uemura, M. J. Org. Chem. 1996, 61, 1375 . 
[12] Burke, M. D.; Schreiber, S. L. Angew. Chem., Int. Ed. 2004, 43, 468.

[13] Lee, J. Y.; Miller, J. J.; Hamilton, S. S.; Sigman, M. S. Org. Lett. 2005, 7, 1837.

[14] (a) Gao, G.; Gu, F. L.; Jiang, J. X.; Jiang, K. Z.; Sheng, C. Q.; Xu, L. W. Chem. Eur. J. 2011, 17, 2698.

(b) Gao, G.; Bai, X. F.; Yang, H. M.; Jiang, J. X.; Lai, G. Q.; Xu, L. W. Eur. J. Org. Chem. 2011, 5039.

(c) Gao, G.; Bai, X. F.; Li, F.; Zheng, L. S.; Zheng, Z. J.; Lai, G. Q.; Jiang, K. Z.; Li, F. W.; Xu, L. W. Tetrahedron Lett. 2012, 53, 2164 .

(d) Zheng, L. S.; Jiang, K. Z.; Deng, Y.; Bai, X. F.; Gao, G.; Gu, F. L.; Xu, L. W. Eur. J. Org. Chem. 2013, 4, 748.

[15] (a) Wu, T. R.; Shen, L. X.; Chong, J. M. Org. Lett. 2004, 6, 2701; (b) Blay, G.; Fernández, I.; Pedro, R. J.; Vila, C. Org. Lett. 2007, 9 , 2601.

(c) Blay, G.; Fernández, M.; Muñoz, C.; Pedro, J. R.; Vila, C. Eur. J. Org. Chem. 2013, 1902

(d) Huang, G. C.; Yin, Z. S.; Zhang, X. G. Chem. Eur. J. 2013, 19, 11992.

[16] (a) Lingenfelter, D. S.; Helgeson, R. C.; Cram, D. J. J. Org. Chem. 1981, 46, 393.

(b) Cox, P. J.; Wang, W.; Snieckus, V. Tetrahedron Lett. 1992, 33, 2253.

(c) Simonsen, K. B.; Gothelf, K. V.; Jørgensen, K. A. J. Org. Chem. 1998, 63, 7536.

(d) Zhang, Z. G.; Dong, Z. B.; Li, J. S. Chirality 2010, 22, 820.

(e) Lin, L.; Zhang, J. L.; Ma, X. J.; Fu, X.; Wang, R. Org. Lett. 2011, 13, 6410 .

[17] Milburn, R. R.; Hussain, S. M. S.; Prien, O.; Ahmed, Z.; Snieckus, V. Org. Lett. 2007, 9, 4403.

[18] (a) Wang, Q.; Chen, S. Y.; Yu, X. Q.; Pu, L. Tetrahedron 2007, 63, 4422.

(b) Yue, Y.; Turlington, M.; Yu, X. Q.; Pu, L. J. Org. Chem. 2009, 74,8681 .

[19] Maruoka, K.; Itoh, T.; Araki, Y.; Shirasaka, T.; Yamamoto, H. Bull. Chem. Soc. Jpn.1988, 8, 2975.

[20] Gribkov, D. V.; Hultzsch, K. C.; Hampel, F. J. Am. Chem. Soc. 2006, 128, 3748.

[21] Kodama, H.; Ito, J.; Hori, K.; Ohta, T.; Furukawa, I. J. Organomet. Chem. 2000, 603, 6 .

[22] Beckendorf, S.; Mancheño, O. G. Synthesis 2012, 2162.

[23] Casas, J.; Baeza, A.; Sansano, J. M.; Nájera, C.; Saá, J. M. Tetrahedron: Asymmetry 2003, 14, 197.

[24] Qin, Y. C.; Liu, L.; Sabat, M.; Pu, L. Tetrahedron 2006, 62, 9335.

[25] Gou, S. H.; Zhou, X.; Wang, J.; Liu, X. H.; Feng, X. M. Tetrahedron Lett. 2008, 64, 2864.

[26] Nájera, C.; Sansano, J. M.; Saá, J. M. Eur. J. Org. Chem. 2009, 2385.

[27] Li, H.; Da, C. S.; Xiao, Y. H.; Li, X.; Su, Y. N. J. Org. Chem. 2008, 73,7398 .

[28] Guo, Q. S.; Liu, B.; Lu, Y. N.; Jiang, F. Y.; Song, H. B.; Li, J. S. Tetrahedron: Asymmetry 2005, 16, 3667.

[29] (a) Matsui, K.; Takizawa, S.; Sasai, H. J. Am. Chem. Soc. 2005, $127,3680$.

(b) Matsui, K.; Tanaka, K.; Horii, A.; Takizawa, S.; Sasai, H. Tetrahedron: Asymmetry 2006, 17,578

[30] Qian, C.; Zhu, C.; Huang, T. J. Chem. Soc., Perkin Trans. 1 1998, 2131.

[31] (a) Vogl, E. M.; Matsunaga, S.; Kanai, M.; Iida, T.; Shibasaki, M. Tetrahedron Lett.1998, 39, 7917.

(b) Matsunaga, S.; Ohshima, T.; Shibasaki, M. Adv. Synth. Catal. $\mathbf{2 0 0 2}, 344,3$.

(c) Shibasaki, M.; Matsunaga, S. J. Organomet. Chem. 2006, 691, 2089.

(d) Shibasaki, M.; Matsunaga, S. Chem. Soc. Rev. 2006, 35, 269.

[32] Hashimoto, T.; Omote, M.; Maruoka, K. Org. Biomol. Chem. 2008, $6,2263$.

[33] (a) Sasai, H.; Arai, R.; Satow, Y.; Houk, K. N.; Shibasaki, M. J. Am. Chem. Soc. 1995, 117, 6194

(b) Sasai, H.; Tokugana, T.; Shizue, W.; Suzuki, T.; Itoh, N.; Shibasaki, M. J. Org. Chem.1995, 60, 7388.

[34] Kadiri, M. Y. E.; Framery, E.; Andrioletti, B. Tetrahedron Lett. 2012, 53, 6335 .

[35] Bunzen, J.; Bruhn, T.; Bringmann, G.; Lützen, A. J. Am. Chem. Soc. 2009, 131, 3621.

[36] Tian, Y.; Chan, K. S. Tetrahedron Lett. 2000, 41, 8813.

[37] Mikami, K.; Ueki, M.; Matsumoto, Y.; Terada, M. Chirality 2001, 13,541 .

[38] Enev, V.; Ewers, C. L. J.; Harre, M.; Nikische, K.; Mohr, J. T. J. Org. Chem. 1997, 62, 7092.

[39] Kiyooka, S.; Tsutsui, T.; Kira, T. Tetrahedron Lett. 1996, 37, 8903.

[40] (a) Marshall J. A. In Comprehensive Organic Synthesis, Vol. 3, Eds.: Trost, B. M.; Fleming, I., Pergamon, Oxford, 1991, p. 975.

(b) Tomooka, K.; Yamamoto, H.; Nakai, T. Liebigs Ann. Recl. 1997, 1275.

[41] Wittig, G.; Löhmann, L. Justus Liebigs Ann. Chem. 1942, 550, 260.

[42] (a) Nakai, T.; Mikami, K. Chem. Rev. 1986, 86, 885.

(b) Kita-mura, M.; Hirokawa, Y.; Maezaki, N. Chem. Eur. J. 2009, $15,9911$.

(c) Sasaki, M.; Ikemoto, H.; Kawahata, M.; Yamaguchi, K.; Takeda, K. Chem. Eur. J. 2009, 15, 4663.

(d) Giampietro, N. C.; Wolf, J. P. Angew. Chem., Int. Ed. 2010, 49, 2922.

[43] Selected examples: (a) Tomooka, K.; Yamamoto, H.; Nakai, T. J. Am.Chem. Soc. 1996, 118, 3317;

(b) Barluenga, J.; Faňanás, F. J.; Sanz, R.; Marcos, C.; Trabada, M. Org. Lett. 2002, 4, 1587.

[44] Santos, L. S. Reactive Intermediates, Wiley-VCH, Weinheim, 2010.

[45] Gao, G. M.S. Thesis, Hangzhou Normal University, Hangzhou, 2012 (in Chinese).

(高广，硕士论文，杭州师范大学，杭州, 2012.)

[46] (a) Somanathan, R.; Flores-Lopez, L.Z.; Montalvo-Gonza-lez, R.; Chavez, D.; Parra-Hake, M.; Auirre, G. Mini-Rev. Org. Chem. 2010, 7, 10 .

(b) Zhong, J. C.; Hou, S. C.; Qian, Q. H.; Yin, M. M.;Na, R. S.; Zheng, B.; Li, Z. Y.; Liu, S. Z.; Wang, M. Chem. Eur. J. 2009, 15, 3069 .

(c) Lombardo, M.; Chia-rucci, M.; Trombini, C. Chem. Eur. J. 2008, 14, 11288 .

(d) Pu, L.; Yu, H. B. Chem. Rev. 2001, 101, 757.

[47] (a) Yoshioka, M.; Kawakita, T.; Ohno, M. Tetrahedron Lett. 1989, 30, 1657.

(b) Takahashi, H.; Kawakita, T.; Yoshioka, M.; Kobayashi, S.; Ohno, M. Tetrahedron Lett. 1989, 30, 7095.

[48] (a) Schmidt, B.; Seebach, D. Angew. Chem., Int. Ed. Engl. 1991, 30, 1321 .

(b) Zhang, F. Y.; Yip, C. W.; Cao, R.; Chan, A. S. C. Tetrahedron: Asymmetry 1997, 8, 585.

(c) Balsells, J.; Walsh, P. J. J. Am. Chem.Soc. 2000, 122, 3250.

(d) Balsells, J.; Walsh, P. J. J. Am. Chem.Soc. 2000, 122, 1802.

(e) Hatano, M.; Miyamoto, T.; Ishihara, K. Adv. Synth. Catal. 2005, 347,1561 . 
(f) Tanaka, T.; Sano, Y.; Hayashi, M. Chem. Asian J. 2008, 3, 1465.

(g) Gou, S. H.; Judeh, Z. M. A. Tetrahedron Lett. 2009, 50, 281.

(h) Zhang, Z. G.; Dong, Z. B.; Li, J. S. Chirality 2010, 22, 820.

(i) Adreu, A. R.; Pereira, M. M.; Bayon, J. C. Tetrahedron 2010, 66, 743.

(j) Von Ronn, R.; Christoffers, J. Tetrahedron Lett. 2011, 67, 334.

[49] (a) Fernández-Mateos, E.; Maciá, B.; Ramón, D. J.; Yus, M. Eur. J. Org. Chem. 2011, 6851.

(b) Fernández-Mateos, E.; Maciá, B.; Yus, M. Eur. J. Org. Chem. 2012, 3732.

(c) Fernández-Mateos, E.; Maciá, B.; Yus, M. Tetrahedron: Asymmetry 2012, 23, 789. (d) Fernández-Mateos, E.; Maciá, B.; Yus, M. Adv. Synth. Catal. 2013, 355, 1249 .

[50] Li, F.; Zheng, Z. J.; Shang, J. Y.; Jiang, K. Z.; Lai, G. Q.; Jiang, J. X.; Xu, L. W. Chem. Asian J. 2012, 7, 2008.

[51] Li, F.; Li, L.; Yang, W.; Zheng, L. S.; Zheng, Z. J.; Jiang, K. Z.; Lu, Y. X.; Xu, L. W. Tetrahedron Lett. 2013, 54, 1584.

[52] Zheng, L. S.; Li, L.; Yang, K. F.; Zheng, Z. J.; Xiao, X. Q.; Xu, L. W. Tetrahedron Lett. 2013, 69, 8777.

[53] Yamamoto, H.; Zhou, F. Synfacts 2014, 10, 56.

[54] Ye, F.; Zheng, Z. J.; Deng, W. H.; Zheng, L. S.; Deng, Y.; Xia, C. G.; Xu, L. W. Chem. Asian J. 2013, 8, 2242.

[55] Song, T.; Zheng, L. S.; Ye, F.; Deng, W. H.; Wei, Y. L.; Jiang, K. Z.; Xu, L. W. Adv. Synth. Catal. 2014, 356, 1708. 